\title{
POSTER
}

\section{Réactivation d'une granulomatose oro-faciale suite à un processus infectieux local}

\author{
Fénelon M, Cambronne C, Catros S, Fricain JC \\ Pole d'odontologie et de santé buccale, CHU de Bordeaux \\ mathildefenelon@live.fr
}

Introduction

Le terme de granulomatose oro-faciale (GOF) a été introduit pour la première fois en 1985 par Wiesenfeld. II s'agit d'une maladie inflammatoire chronique affectant les tissus mous de la sphère maxillo-faciale. Cliniquement la GOF se caractérise le plus souvent par un œdème labial récurrent ou persistant ainsi que par des manifestations intra orales à type d'ulcération ou d'hyperplasie affectant principalement la gencive et la langue (Al Johani K A et al. J Am Acad Dermatol, 62:611-20). Le diagnostic repose sur l'observation d'une lésion granulomateuse non caséeuse à l'examen histologique en l'absence d'une affection systémique concomitante. L'étiologie de la GOF reste incertaine bien qu'une origine allergique, infectieuse ou encore une prédisposition génétique ait été évoquée (Grave $B$ et al. Oral diseases, 15, 46-51).

\section{Patient}

Le cas d'une patiente de 61 ans est rapporté. La patiente consultait pour des douleurs gingivales évoluant depuis trois mois en rapport avec la mise en place d'une prothèse amovible totale fixée sur deux implants. II s'agissait d'une patiente présentant une polyarthrite rhumatoïde traitée par Planquenile. Un an auparavant, elle avait consulté au sein du Service de Dermatologie pour une tuméfaction labiale supérieure pour laquelle le diagnostic de macrochéilite de Miescher avait été posé et qui s'était résolu spontanément au bout de six mois. Des examens complémentaires fibroscopique et coloscopique et un scanner thoraco-abdomino-pelvien avaient permis d'éliminer une maladie de Crohn et une sarcoïdose.

Lors de cette nouvelle consultation, l'examen clinique mettait en évidence une macrochéilite labiale inférieure ainsi qu'une importante inflammation gingivale en regard de la prothèse. Un processus infectieux avec suppuration était également observé en regard de cette prothèse. La prothèse a été sectionnée afin d'être déposée et une biopsie gingivale a été réalisée. Des antalgiques de pallier 1 associées à des bains de bouche pluriquotidiens ont été prescris. L'analyse histologique mettait en évidence une lésion granulomateuse épithélioïde gigantocellulaire. Une semaine après la dépose de la prothèse, la patiente ne présentait plus de douleur, et trois semaines après, on observait une régression complète de la macrochéilite. 
Discussion

L'œdème labial est la symptomatologie la plus fréquente de la GOF. Les manifestations intra orales sont multiples. Le diagnostic différentiel est important car la GOF peut être une manifestation buccale d'une affection systémique (maladie de Crohn, sarcoïdose, tuberculose). À notre connaissance, il n'a jamais été décrit dans la littérature de réactivation de la GOF par un processus infectieux local.

Conclusion

La GOF représente souvent un dilemme diagnostique pour le praticien. La démarche repose sur un diagnostic d'élimination (F Alawi. Dent Clin N Am, 57 :657-671). 